\title{
Cinétique d'écoulement du latex d'Hevea brasiliensis après saignée
}

\author{
Flow kinetics of latex tapped from "Hevea brasiliensis"
}

\author{
Claude Lioret ${ }^{*}$, Daniel Ribaillier** et Jean-Claude Combe** \\ * Professeur de Physiologie Végétale à l'Université de Paris Sud (Orsay) \\ ** Institut de Recherche sur le caoutchouc en Afrique
}

\section{Introduction}

La production de caoutchouc naturel est essentiellement obtenue à partir de l'Hevea brasiliensis, arbre appartenant à la famille des Euphorbiacées et cultivé, dans les régions équatoriales chaudes et humides, en plantations de plusieurs milliers d'hectares.

Le caoutchouc est un composant du latex, liquide biologique contenu dans le système laticifère constitué de vaisseaux anastomosés et localisé dans l'écorce du tronc, des branches et des racines.

Le latex est récolté à partir d'une incision de l'écorce (saignée), sectionnant les vaisseaux laticifères. Le latex. s'écoule de cette section, selon une cinétique dont l'analyse fait l'objet du présent article. La compréhension et si possible la maîtrise du phénomène sont très importantes pour l'exploitation. Un écoulement peu abondant foumit une production insuffisante, un écoulement trop abondant peut épuiser l'arbre.

\section{Le système laticifère}

Ce système est composé de conduits (vaisseaux laticifères) d'un diamètre d'environ $20 \mu$ localisés dans le tissu liberien (phloème). Ces vaisseaux résultent de la jonction, par disparition des parois transversales, de cellules issues du cambium. Ce dernier a un fonctionnement rythmique. Les laticifères issus d'une phase de ce fonctionnement sont groupés en un "manteau" au sein duquel les conduits s'anastomosent. Les différents manteaux sont concentriques; les plus jeunes sont proches du cambium, les plus anciens, qui ne sont plus fonctionnels, sont rejetés vers l'extérieur et disparaissent dans un tissu scléreux (Fig. 1). Le nombre de manteaux fonctionnels (chacun comprenant de 2 à 3 rangées de vaisseaux laticifères) est compris entre 10 et 20 pour un arbre d'une dizaine d'années. Il ne parait pas exister d'anastomoses entre vaisseaux appartenant à des manteaux différents. Le nombre de laticifères fonctionnels,

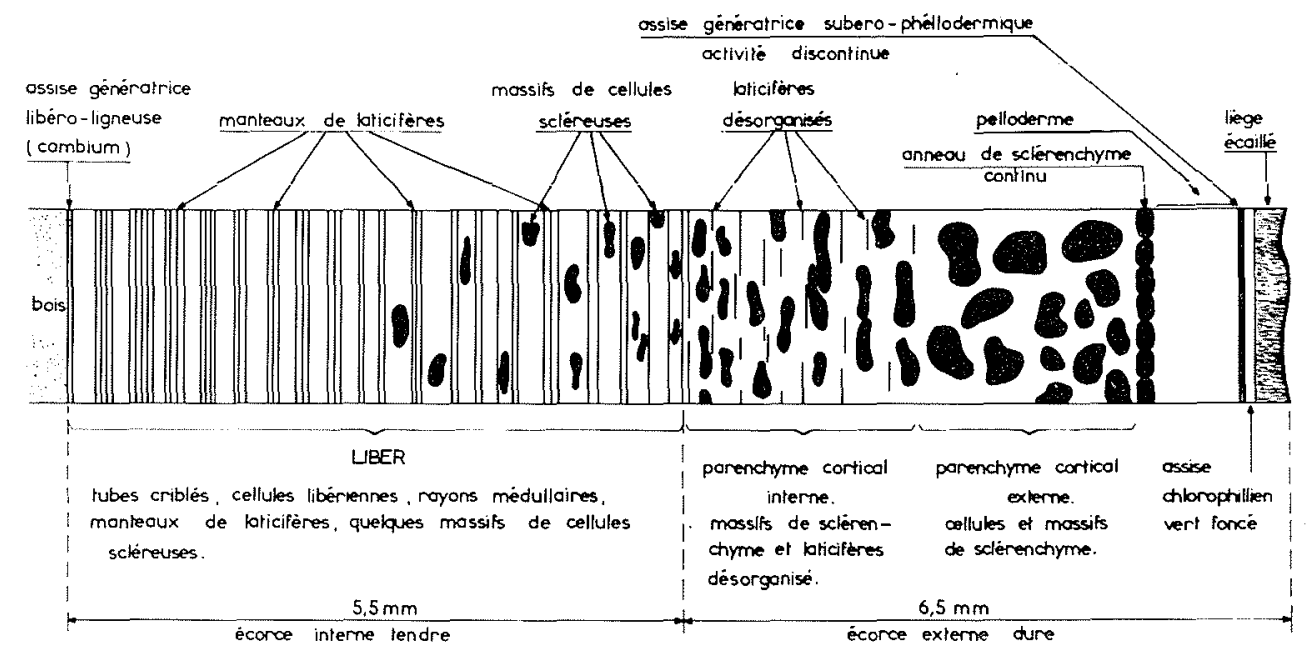

Figure 1 - Représentation schématique de l'écorce vierge (n'ayant jamais été soumise à la saignée) d'un Hevea de 20 ans (coupe radiale). Tirée de Bouychou (1962). 
pour une circonférence (à $1 \mathrm{~m}$ du sol) du tronc d'un arbre de 8 ans, se situe autour de 8000 .

\section{La saignée}

Elle consiste à pratiquer, avec une gouge, une incision de l'écorce sectionnant les conduits fonctionnels. La profondeur de l'incision est généralement inférieure à $1 \mathrm{~cm}$ pour ne pas léser le cambium. L'incision fait un angle d environ $20^{\circ}$ avec l'horizontale ; elle constitue une spirale dite entière (S) si elle fait le tour complet de l'arbre, ou d'une fraction de spirale $(\mathrm{S} / 2, \mathrm{~S} / 4)$ selon les modalités de l'exploitation. En règle générale, un arbre est saigné 2 fois par semaine avec des intervalles de saignée $(3 / 4$, ou $5 / 2)$ variant également selon les types d'exploitation. Du fait des saignées antérieures, l'opération consiste en fait, à procéder à l'ablation d'une pellicule, d'épaisseur inférieure à $1 \mathrm{~mm}$, à la surface de la section précédente dite encoche. La hauteur de l'encoche s'abaisse ainsi progressivement à chaque opération, tandis que le cambium regénère l'écorce des parties supérieures éliminée par les prélèvements précédents. Le latex s'écoule par pesanteur le long de cette encoche et est recueilli dans une tasse ou dans un sac plastique à son extrémité.

On recueille de 150 à $300 \mathrm{ml}$ de latex par arbre et par saignée.

\section{La cinétique d'écoulement}

Les figures 2 et 3 représentent l'évolution du débit $(V)$ en fonction du temps. C'est la récolte cumulée qui est mesurée. Soient $q i$ la quantité recueillie au temps $t i$ et $q j$ celle recueillie au temps $t j$ on a $V i j=\frac{q j-q i}{t j-t i}$. Les mesures ont été réalisées toutes les $1 / 2$ minutes au début de l'écoulement puis pour des intervalles de temps plus grands ( $1 \mathrm{~min}, 2 \mathrm{~min}, 5 \mathrm{~min}$ ) lorsque l'écoulement se ralentit.

Les deux courbes, obtenues à partir d'arbres appartenant à deux clônes ${ }^{(1)}$ différents, ont des profils comparables, marqués par:

a) une chute très rapide du débit pendant les premières minutes suivant la saignée ;

b) ensuite, une diminution progressive et plus lente de ce débit avec de nombreuses irrégularités;

c) en fin d'écoulement un débit toujours décroissant très faible et régulier finissant par s'annuler.

La figure 2 concerne le clône PR 107, le débit initial est élevé (17 $\mathrm{ml} / \mathrm{min})$, la durée đ'écoulement relativement courte $(300 \mathrm{~min})$. La figure 3 , relative au clône RRIM 501 montre une cinétique caractérịsée par un débit initial plus faible $(5,3 \mathrm{ml} / \mathrm{min})$ avec une durée d'écoulement près de 2 fois plus longue (550 $\mathrm{min})$.

Paardekooper et Samosorn (1969), Milford et al. (1969) ont proposé le rapport $\frac{\text { débit initial }}{\text { quantité totale obtenue }}$ (nommée "plugging index") comme un indice définissant les propriétés d'écoulement des différents clônes.

Les figures $4,5,6$ et 7 représentent les courbes obtenues après transformations des données réalisées dans lespérance d'obtenir des régressions linéaires.

(1) clône $=$ ensemble d'individus issus par reproduction végétative (non sexuée) d'un même géniteur et ayant de ce fait le même patrimoine génétique.

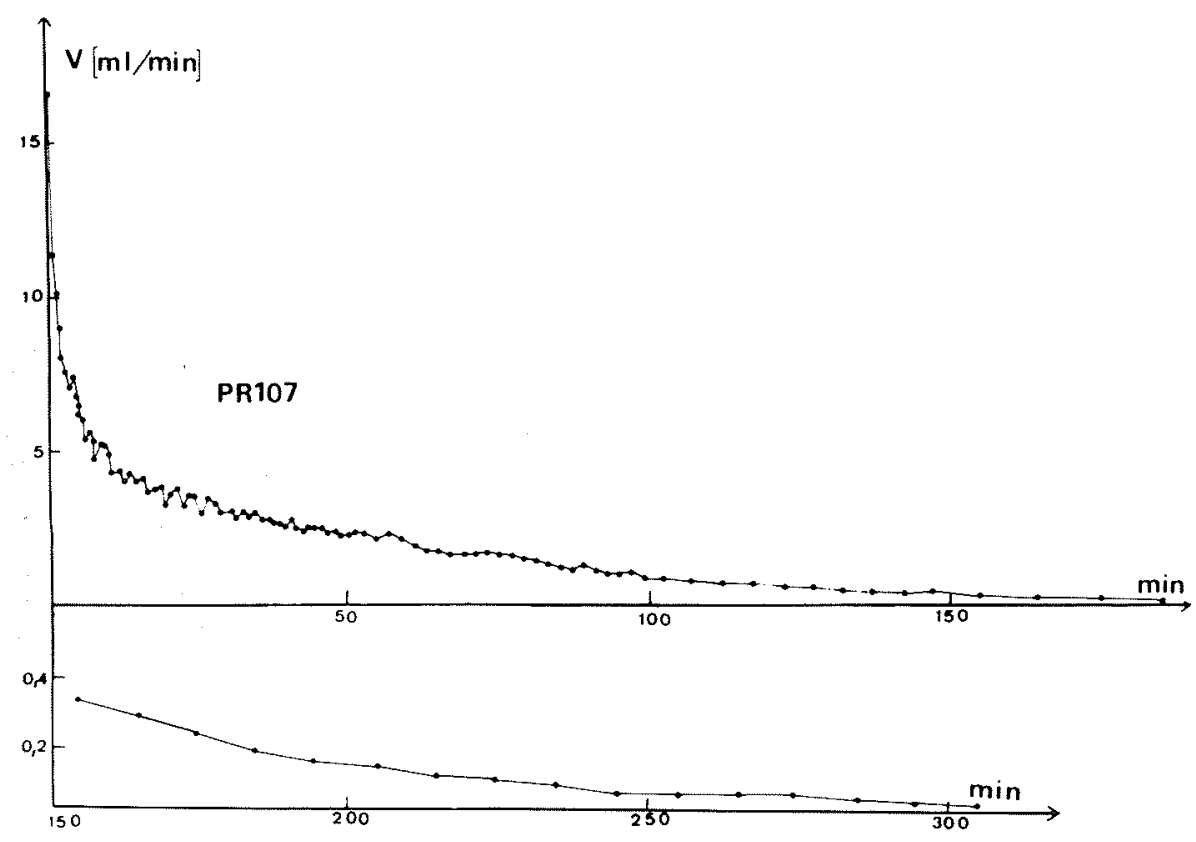

Figure 2 - Cinétique d'écoulement du latex d'un arbre appartenant au clone PR 107, saigné en spirale entière. 


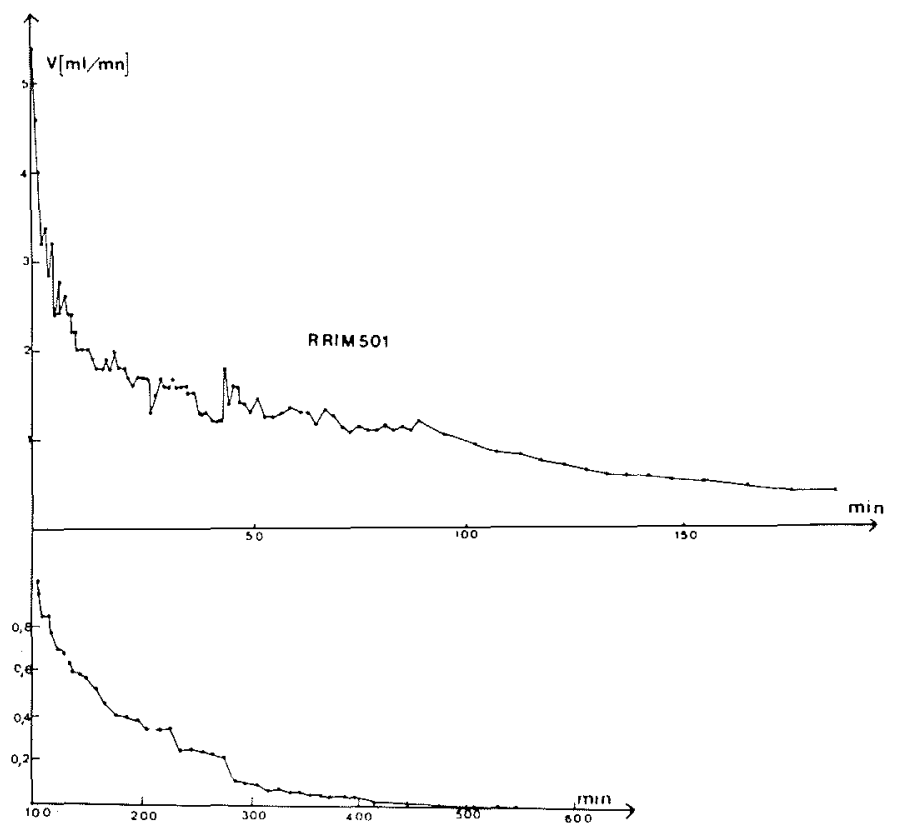

Figure 3 - Cinétique d'écoulement du latex d'un arbłe appartenant au clone RRIM, stigné en spirale entière.

Paardekooper et Samosorn (1969), Ninane (1970) ont proposé le modèle exponentiel $\left(V=V o e^{-k t}\right)$ comme rendant compte de la globalité du phénomène. La figure 4 montre que ce modèle peut s'appliquer pour la partie médiane de l'écoulement mesuré avec un arbre du clone PR 107, mais ne peut être extrapolé pour les parties initiales et finales. Pour le clone RRIM 507 (Fig. 5), l'assimilation est plus discutable. Nous avons réalisé de nombreux essais de telles régressions sans obtenir de résultats réellement satisfaisants. Pour un même clone, nous avons obtenu de meilleurs coefficients de corrélation parfois avec la transformation $\log V=f(t)$ parfois avec la transformation $\sqrt{v}=f(t)$.

\section{Interprétation des phénomènes}

\section{a) Expulsion par détente élastique}

Buttery et Boatman (1966, 1967), Ninane (1970) ont mesuré, par implantation dans l'écorce d'une aiguille creuse prolongée par un tube capillaire fermé, la pression

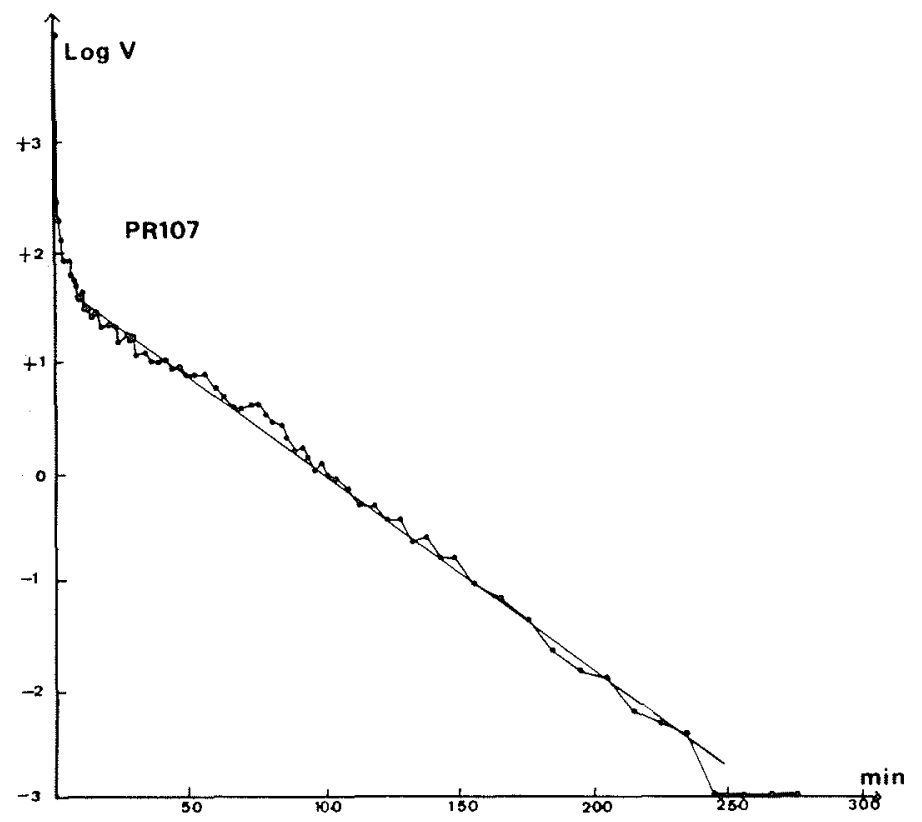

Figure 4 - Représentation de la relation $\log V=f$ (temps) d'après les données de la figure 2 (clone PR 107). 


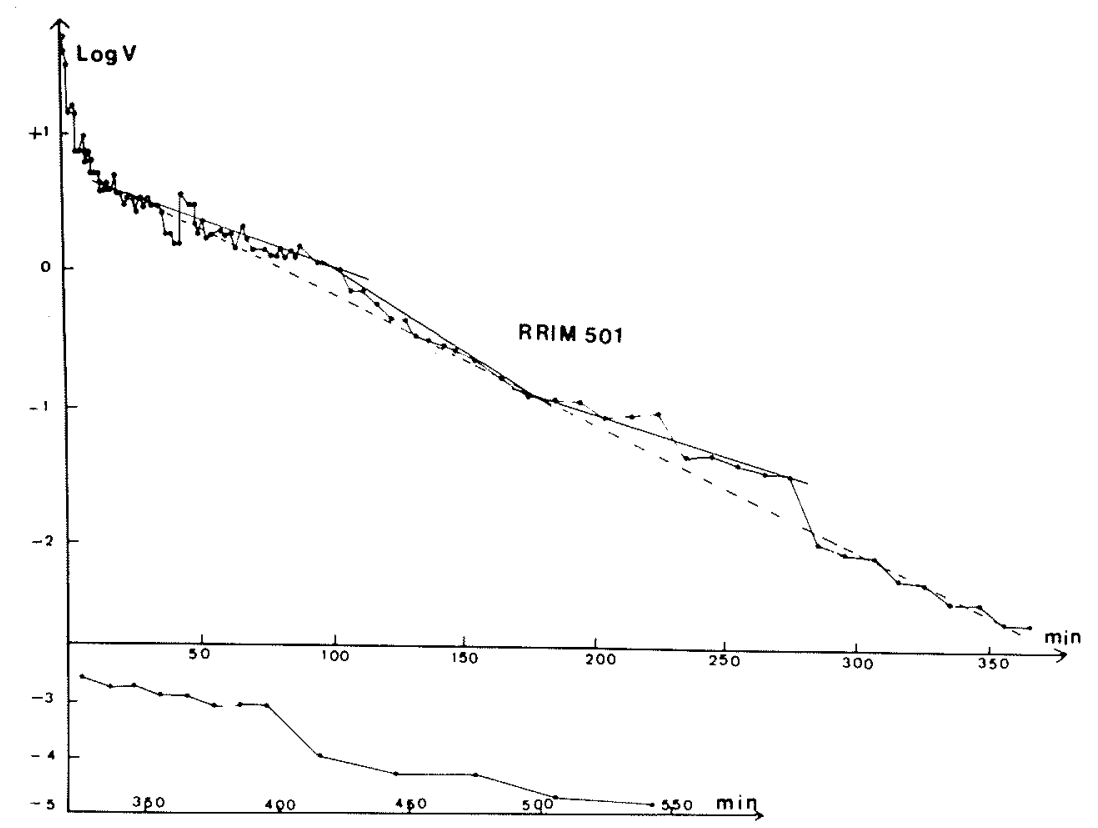

Figure 5 - Représentation de la relation $\log V=f$ (temps) d'après les données de la figure 3 (clone RRIM 501).

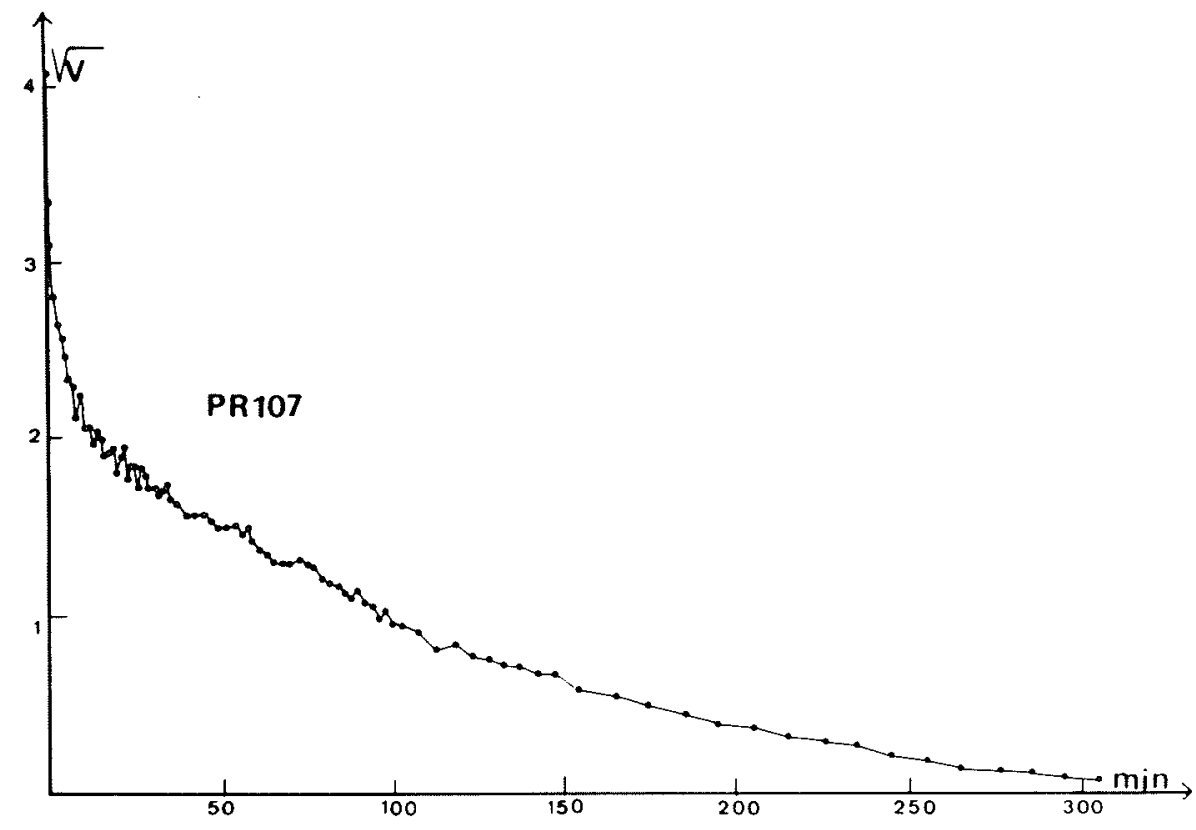

Figure 6 - Représentation de la relation $\sqrt{V}=f$ (temps) d'après les données de la figure 2.

mécanique à l'intérieur des laticifères: le latex pénétrant dans l'aiguille comprime l'air contenu dans le tube, la longueur de la partie remplie d'air est proportionnelle à la pression subie par le latex. Cette technique a montré que cette pression (dite de turgescence) est assez élevée (plus de 10 atmosphères, parfois 16). Les parois des cellules et des vaisseaux laticifères sont légèrement élastiques et se trouvent tendues sous l'effet de cette pression.

La saignée porte brutalement le contenu des laticifères à la pression atmosphérique. La figure 8 montre les mesures obtenues par Buttery et Boatman (1967) : la chute de pression est très nette pour la zone proche de l'encoche, elle est moins accentuée pour les régions plus profondes. Les cellules contiguës aux vaisseaux ne sont pas soumises à cette dépression, et tendent à comprimer ces derniers. Cette compression par les cellules voisines, jointe à la diminution de la tension de la paroi élastique, expulse une partie du contenu des laticifères. Cette interprétation a été proposée pour la première fois par Freywyssling (1932), reprise par divers auteurs dont Riches et Gooding (1952).

La transformation $1 / V=f(t)$ met systématiquement en évidence une cassure après quelques minutes d'écou- 


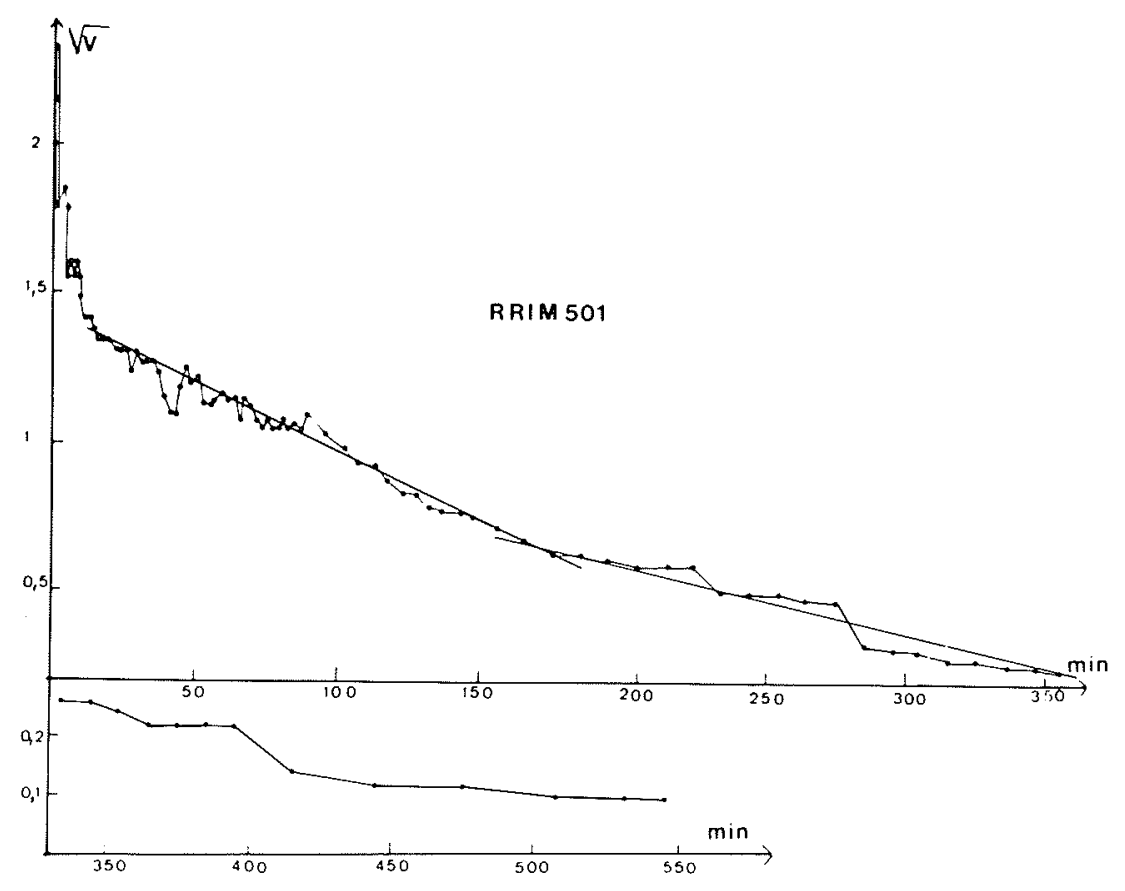

Figure 7 - Représentation de la relation $\sqrt{V}=f$ (temps) d'après les données de la figure 3.

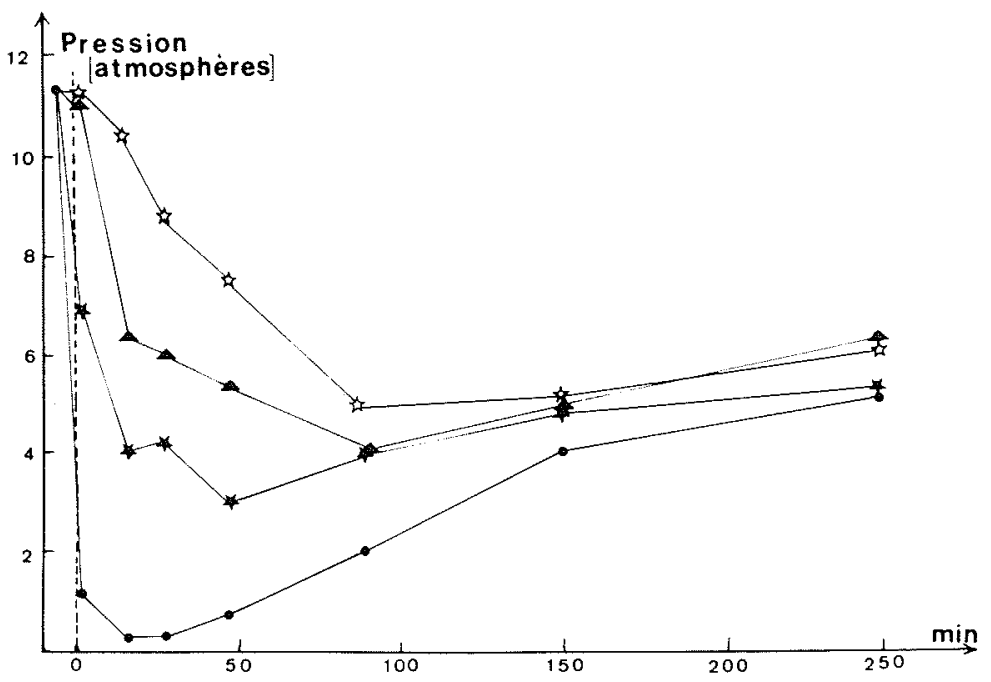

Figure 8 - Variations, après la saignée (marquée par la ligne verticale pointillée), de la pression de turgescence à $4 \mathrm{~cm}$ (cercles noirs) $32 \mathrm{~cm}$ (étoiles noires), $62 \mathrm{~cm}$ (triangles noirs) et $123 \mathrm{~cm}$ (étoiles claires) en dessous de l'encoche, pour un arbre ayant déjà subi 16 saignées. Données de Buttery et Boatman (1967).
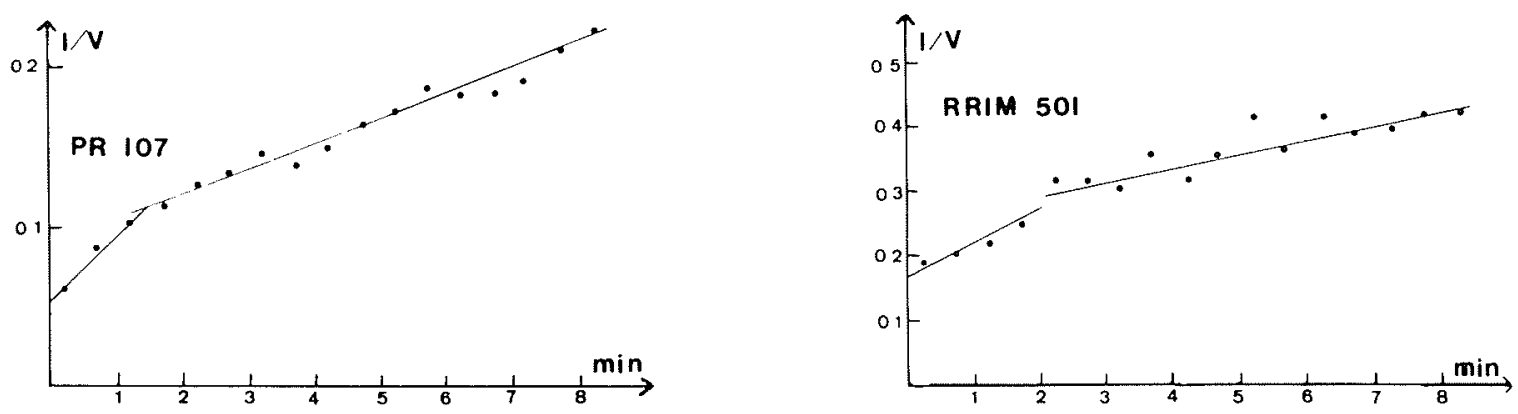

Figure 9 - Représentation, pour le début de l'écoulement, de la relation $1 / V=f$ (temps) d'après les données des figures 2 et 3. 
lement (Fig. 9). Il est probable que ce phénomène d'expulsion par détente élastique corresponde à cette période relativement brève suivant la saignée.

$A$ ce phénomène est liée une diminution du diamètre du tronc, mesuré avec des dendromètres très sensibles (Gooding, 1951 ; Boatman, 1966 ; Ninane, 1970). La diminution est de l'ordre de 25 à $50 \mu$ pour un tronc, ce qui correspond à moins d'1 $\mu$ par laticifère (environ 50 vaisseaux laticifères sur un diamètre). Frey. Wyssling (1932) supposait un applatissement total des conduits sous l'effet de la pression exercée par les autres cellules. Ces dernières données ne permettent pas une telle interprétation.

\section{b) Arrivée dans les laticifères de matériel provenant des autres tissus de l'arbre.}

Si le volume diminue si peu, il est évident que le matériel expulsé est remplacé.

Les auteurs précités (Frey-Wyssling, Riches et Gooding) estimaient que le matériel de remplacement était simplement l'eau diffusant à travers les membranes $\mathrm{du}$ fait du fort abaissement de potentiel hydrique du latex résultant lui-même de l'effondrement de la pression de turgescence. S'il en est ainsi, on doit s'attendre à une dilution du latex recueilli au fur et à mesure de l'écoulement.

Le tableau I montre qu'il en est bien ainsi pour le caoutchouc, mais que cela ne se produit pas pour les glucides ou les protéines. Le matériel nouveau est de type protoplasmique et provient des cellules voisines vraisemblablement au niveau des plasmodesmes, fins canalicules d'un diamètre d'environ $0,1 \mu$ traversant les parois, permettant des communications entre différentes cellules d'une part et cellules et vaisseaux d'autre part. une transpiration abondante de l'arbre: l'appel d'eau au niveau foliaire se répercute de proche en proche dans les différents organes et supprime la différence de pression existant entre tissus et laticifères. De même, les Heveas étant des arbres à feuilles caduques, l'exploitation doit être arrêtée lors de la refoliation, le flux d'eau et de matières est alors capté pour la formation de nouvelles feuilles et n'alimente plus les vaisseaux laticifères.

Les différents auteurs qui ont étudié ce problème ont admis que l'écoulement suivait la loi de Poiseuille, le latex étant considéré comme un liquide newtonien. Ce point n'est pas évident, il s'agit d'un liquide biologique complexe, comportant de nombreux éléments figurés en suspension, très instable et susceptible de subir des transformations importantes (dont la principale est la coagulation) au cours des manipulations. Il est difficile dans ces conditions d'effectuer une étude expérimentale sur le latex recueilli.

$\mathrm{Si}$ les phénomènes incriminés étaient seuls en cause, l'écoulement s'arrêterait lorsque la đifférence de pression s'annule, le système se retrouvant à la pression atmosphérique. Frey-Wyssling (1939), Riches et Gooding (1952) ont interprété la diminution lente du débit par l'augmentation $\mathrm{du}$ paramètre "longueur parcourue" intervenant dans la loi de Poiseuille, le latex recueilli en fin d'écoulement provenant des régions les plus éloignées de l'encoche.

\section{c) Obturation des laticifères}

Or la pression remonte (cf Fig. 8). Cette remontée résulte, en plus de l'arrivée de nouveau matériel, d'une obturation de la section. Boatman (1966), le premier, a montré que, si au cours de l'écoulement on rénove l'encoche par ablation d'une très faible épaisseur de

\begin{tabular}{|c|c|c|c|}
\hline \multicolumn{2}{|c|}{ Tableau I - Variation de composition du latex au cours de l'écoulement (Données de Ribaillier, 1972) } \\
\hline Fractions ${ }^{\circ}$ & $\begin{array}{c}\text { Teneur en caoutchouc } \\
\text { sec (DRC) } \\
\text { (en\% du latex) }\end{array}$ & $\begin{array}{c}\text { Glucides } \\
\text { (en mg d'hexoses } \\
\text { par ml de latex) }\end{array}$ & $\begin{array}{c}\text { Azote } \\
\text { protéique } \\
\text { (mg/ml de latex) }\end{array}$ \\
\hline 1 & 47,1 & 10 & 10 \\
2 & 42,3 & 9,4 & 10,3 \\
3 & 40,1 & 7,8 & 9,7 \\
4 & 38.4 & 8,3 & 10,3 \\
5 & 37,1 & 9,3 & 10,2 \\
6 & 35,7 & 11,2 & 10,3 \\
7 & 35,1 & 13,2 & 10,4 \\
8 & 34,8 & 14,3 & 10,1 \\
9 & 34,7 & 15,1 & 10,1 \\
\hline
\end{tabular}

Le moteur de cette arrivée de matériel est la différence de pression existant entre conduits et cellules. Le diamètre très fin des plasmodesmes offre une résistance importante qui prolonge la différence de pression. S'il existe une pression de turgescence faible dans les tissus autres que les laticifères, l'écoulement s'arrête rapidement. Ninane (1970) a bien montré que cet écoulement était très limité lors de conditions climatiques favorisant celle-ci $(<1 \mathrm{~mm})$, on observe une augmentation immédiate du débit. Celui-ci diminue ensuite progressivement. $\mathrm{Ce}$ phénomène est répétable et l'on peut procéder à plusieurs rénovations successives (Fig. 10).

Ces faits montrent d'une part que l'obturation est un phénomène assez rapide, d'autre part qu'elle est très superficielle. Ceci est fort heureux car si elle se faisait en profondeur, l'exploitation ne serait pas possible. 


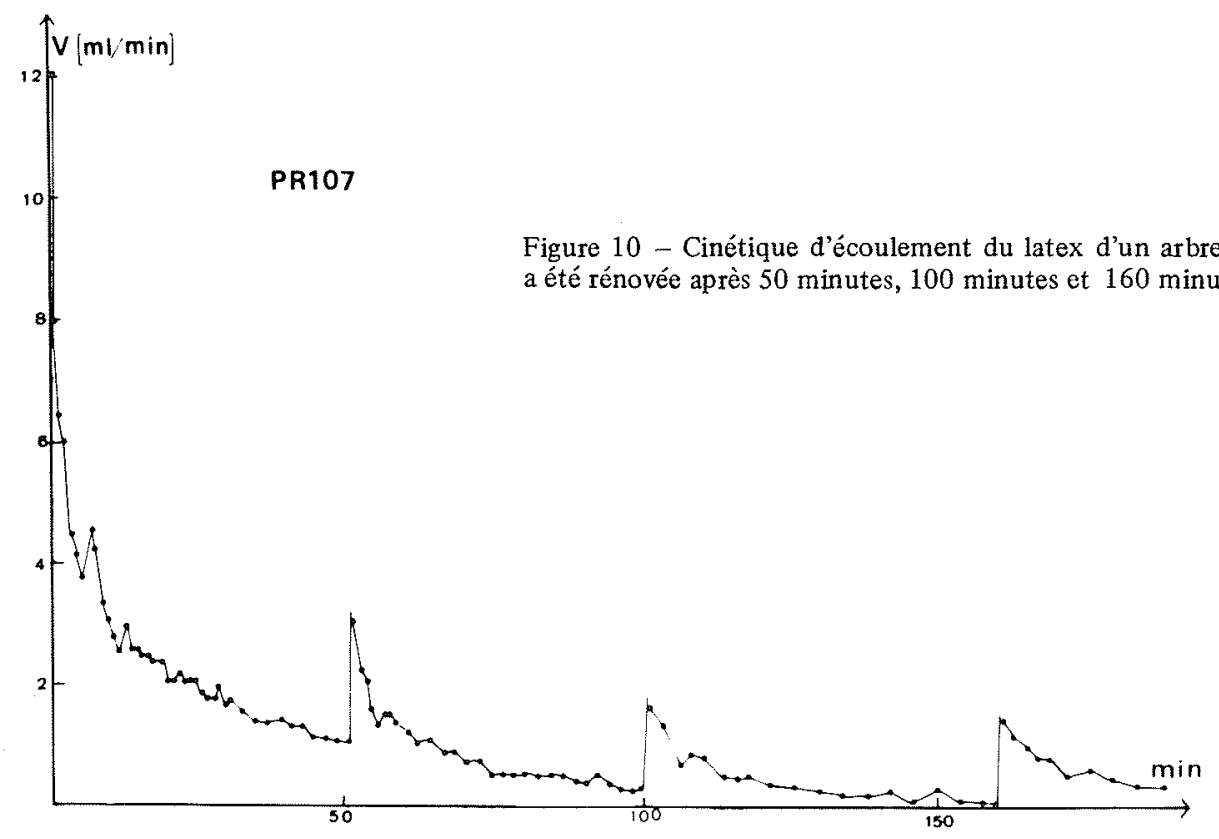

L'obturation résulte de la coagulation du latex dont nous ne discuterons pas ici du mécanisme (cf sur ce sujet Hanower et al., 1976). Les coagulums de dimensions supérieures à un certain seuil vont se coincer à l'orifice des vaisseaux, les interstices éventuels doivent être très rapidement colmatés par ceux de plus petite taille. La probabilité d'obturation d'un laticifère doit dépendre : d'une part de la distribution statistique des coagulums en fonction de leurs dimensions ; d'autre part de l'intensité de forces d'expulsion faisant intervenir la vitesse découlement et les différences de pression (qui se restaurent lorsqu'un conduit est bouché). Les importantes irrégularités observées sur les courbes cinétiques sont interprétées par des obturations temporaires, les bouchons formés étant expulsés plus ou moins rapidement.

Le traitement de l'écorce située sous l'encoche par 1" "Ethrel" (produit dont la décomposition libère de l'ethylène) est suivi d'une diminution très marquée des propriétés coagulantes du latex et permet des récoltes beaucoup plus abondantes (cf. Ribaillier, 1972, Hanower et al., 1976).

\section{Conclusion}

Ce bref exposé tend à montrer que le processus apparemment simple qu'est l'écoulement d'un liquide biologique après blessure superficielle d'un organisme végétal fait intervenir pour sa compréhension, en plus de paramètres physiques simples, de nombreux aspects de la physiologie de cet organisme. Ont été ainsi évoqués l'équilibre hydrique de l'arbre, le passage de matières entre différents tissus, le processus de coagulation (faisant lui même intervenir la composition chimique, la répartition de divers constituants entre différentes phases) etc... L'exemple illustre comment la connaissance physiologique est utile à une production agricole d'une matière première industrielle.

\section{Bibliographie}

BOATMAN S.G. (1966). - Preliminary physiological studies on the promotion of latex flow by plant growth substances. J. Rubb. Res. Inst. Malaya, 19, 243-258.

BOUYCHOU J.G. (1962) - Manuel du planteur d'Hevea.SETCO édit. Paris.

BUTTERY B.R. et BOATMAN S.G. (1966) - Manometric measurement of turgor pressures in laticiferous phloem tissues. J. Exp. Bot., 17, 283-296.

BUTTERY B.R. et BOATMAN S.G. (1967) - Effects of tapping, wounding, and growth regulators on turgor pressure of Hevea brasiliensis Müil. Arg. J. Exp. Bot., 18, 644-659.

FREY-WYSSLING A. (1932) - Investigations in the dilution reaction and the movement of the latex of Hevea brasiliensis during tapping. Arch. Rubbercuet, 16, 285-327.

GOODING E.G.B. (1952) - Studies in the physiology of latex.II latex flow on tapping Hevea brasiliensis : associated changes in trunk diameter and latex concentration. New. Phytol., 51, 11-29.

HANOWER P., BRZOZOWSKA J. et LIORET C. (1976) Etude du mécanisme de la coagulation du latex d'Hevea brasiliensis (Kunth) Müll. Arg. I. Facteurs agissant sur la coagulation. Physiol: végét., 14, 677-693.

MILFORD G.E.F. et PAARDEKOOPER E.C. (1969) - Latex Vessel plugging, its importance to yield and clonal behaviour. J. Rubb. Res. Instit. Malaya, 21, 274-282.

NINANE F. (1970) -- Les aspects écophysiologiques de la productivité chez Hevea brasiliensis Mül. Arg., au Cambodge. These Doctorat, Université de Louvain (309 p.).

PAARDEKOOPER E.C. et SAMOSORN S. (1969) - Clonal variation in latex flow pattern. $J$. Rubb. Res. Instit. Malaya, $21,264-273$.

RIBAILLIER D. (1972) - Quelques aspects du rôle des lutoides dans la physiologie et l'écoulement du latex d'Hevea brasiliensis (Kunth) Müll. Arg. Action de produits libérant de l'éthylène. Thèse de Doct. Sc. Nat., Université d'Abidjan (181 p).

RICHES J.P. et GOODING E.G.B. (1952) - Studies in the physiology of latex. I Latex flow on tapping, theoritical considerations. New Phytol., 51, 1-10. 


\section{Discussion}

Président : M.S. HENIN

Le Président remercie M. LIORET de son exposé et ouvre la discussion.

A une question de M. VERNET, M. LIORET répond en indiquant que les courbes d'écoulement présentées ont toujours été obtenues en plein air, sur des arbres de plantations.

M. VERNET lui demande alors comment il distingue les effets de la transpiration sur la réduction du potentiel?

M. LIORET répond que NINANE, qui a travaillé à l'Institut de recherches sur le caoutchoux au Cambodge, a mesuré simultanément la production et la pression de turgescence. Il a constaté une corrélation étroite entre baisse de production, baisse de turgescence et conditions climatiques favorisant la transpiration (vent, fort éclairement, hygrométrie faible). L'interprétation la plus satisfaisante est celle-ci : un pouvoir évaporant de l'atmosphère entraîne une transpiration élevée, laquelle provoque l'abaissement de la pression de turgescence facteur direct de l'écoulement d'où baisse de production.

M. BONNEMAIN se dit "intrigué par l'augmentation de la teneur en glucide, observée peu après l'incision". Il demande à M. LIORET s'il a une explication à ce sujet.

M. LIORET n'a aucune idée.

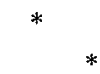

Le Président clôt les débats par une brève allocution :

Je voudrais, Messieurs, dire quelque mots pour terminer. D'abord pour remercier les orateurs et les intervenants. Il y a là une première prise de contact qui sera probablement à développer dans l'avenir, ainsi que le disait le Président CAZENAVE au début de cette séance.

J'ai relevé un élément très intéressant. Si l'on prend un sol, la quantité d'eau qui s'évapore est de 30 à $40 \mathrm{~mm}$ puis l'évaporation devient très lente : le milieu s'est désséché et les couches profondes d'eau ne sont pas exploitées. Avec un végétal, pour arriver à la même situation, on va évaporer 200 ou $250 \mathrm{~mm}$ d'eau. Ainsi et en dépit de tout ce que nous avons vu, la fonction de transfert dans un végétal est plus efficace qu'une masse de matériaux meubles empilés sur eux-mêmes. Et pourtant nous avons vu l'éxtraordinaire complexité du problème.

L'hydrodynamicien se réfère à des lois fondamentales, qui d'ailleurs ne sont pas toujours très faciles à appliquer parce qu'elles se compliquent quand il s'agit d'appareils dans lesquels l'eau circule à travers des circuits complexes; néanmoins il y a un certain nombre de situations qui posent moins de problèmes que les autres, parce que le liquide ne fait pas partie de la structure du matériau c'est-à-dire, du filtre dans lequel il s'écoule.

Je pense qu'il serait intéressant de rapprocher certains points de vue et, peut-être aussi, les terminologies. Par exemple, dans ce que disait M. CRUIZIAT à propos du fonctionnement des réserves, je crois que le phénomène qui a été mis en évidence au départ s'apparente à la consolidation de couches d'argile soumise à une pression agissant sur une surface. On pourrait retrouver des dispositions similaires en biologie et se rapprocher de certains concepts utilisés par les hydrodynamiciens ou les mécaniciens du sol. Je pense en particulier à la pression de turgescence : la cellule gonfle parce qu'elle est dans l'eau, ensuite elle va relâcher l'eau, mais où l'eau va-t-elle passer? Autrement dit, il y a un certain nombre de mécanismes qui sont peut-être examinés de façon un peu gratuite, et je regrette que nous n'ayons pas eu suffisamment de temps pour les approfondir.

Depuis le schéma volontairement simpliste qui a été présenté par M. BERGER jusqu'au schéma très complexe correspondant à la circulation des liquides biologiques, nous constatons que la complexité croissante des systèmes résulte de la prise en compte de propriétés de plus en plus variées concernant soit le fluide soit la structure à travers laquelle il s'écoule.

C'est ainsi que pourraient se faire certaines confrontations d'idées et une comparaison de la terminologie. Ceci permettrait aux uns de mieux profiter des données fondamentales, et aux autres de mieux comprendre les démarches suivies par d'autres spécialistes.

Je laisse la parole au Président CAZENAVE pour clore cette journée qui a été très instructive.

Monsieur CAZENAVE conclut, comme suit, la Session :

Je prendrai la parole essentiellement pour remercier et le Président de séance et l'auditoire et pour dire que si vous éprouvez le sentiment que certains sujets évoqués ici ont été un peu limités par le temps et que certaines réflexions complémentaires pourraient être faites, la Société Hydrotechnique de France est toujours prête à servir de lieu d'accueil et à contribuer à des groupes de travail. Nous avons la logistique et les animateurs et nous les mettrons très volontiers à la disposition de ceux qui voudraient aborder tous les domaines qui touchent à l'eau.

Par conséquent, s'il y avait un dernier mot à dire, ce serait "au revoir et merci" plutôt que "adieu". Nous espérons revoir souvent les biologistes et les spécialistes du milieu végétal parmi nous. 